\title{
Maximum Angular Separation Epochs for Exoplanet Imaging Observations
}

\author{
Stephen R. Kane ${ }^{1}(\mathbb{1})$, Tiffany Meshkat ${ }^{2}$ (1) , and Margaret C. Turnbull $^{3}$ \\ ${ }^{1}$ Department of Earth Sciences, University of California, Riverside, CA 92521, USA; skane@ucr.edu \\ 2 IPAC, Caltech, M/C 100-22, 1200 East California Boulevard, Pasadena, CA 91125, USA \\ ${ }^{3}$ SETI Institute, Carl Sagan Center for the Study of Life in the Universe, Off-Site: 2801 Shefford Drive, Madison, WI 53719, USA \\ Received 2018 August 28; revised 2018 October 12; accepted 2018 October 16; published 2018 November 15
}

\begin{abstract}
Direct imaging of exoplanets presents both significant challenges and significant gains. The advantages primarily lie in receiving emitted and, with future instruments, reflected photons at phase angles not accessible by other techniques, enabling the potential for atmospheric studies and the detection of rotation and surface features. The challenges are numerous and include coronagraph development and achieving the necessary contrast ratio. Here, we address the specific challenge of determining epochs of maximum angular separation for the star and planet. We compute orbital ephemerides for known transiting and radial velocity planets, taking Keplerian orbital elements into account. We provide analytical expressions for angular star-planet separation as a function of the true anomaly, including the locations of minimum and maximum. These expressions are used to calculate uncertainties for maximum angular separation as a function of time for the known exoplanets, and we provide strategies for improving ephemerides with application to proposed and planned imaging missions.
\end{abstract}

Key words: planetary systems - techniques: radial velocities - techniques: high angular resolution

\section{Introduction}

Direct imaging of exoplanets provides opportunities for significantly extending exoplanet science, such as direct atmospheric retrieval (Feng et al. 2018), and unlocking intrinsic planet properties, such as albedo, rotation, and obliquity (Cowan et al. 2009; Kane \& Torres 2017). The method of direct imaging also remains one of the most challenging techniques for studying exoplanets. At the present time, only $\sim 1 \%$ of the known exoplanets have been discovered using direct imaging, according to data from the NASA Exoplanet Archive (Akeson et al. 2013). However, many technology advancements, both instrumental and with software, have taken place over recent years that allow significantly enhanced capabilities to extract a planetary signature from the stellar diffraction pattern. The Gemini Planet Imager (Macintosh et al. 2014) and the Spectro-Polarimetric High-Contrast Exoplanet Research (Beuzit et al. 2008) instruments have contributed significantly to the ground-based imaged planets inventory. Examples of other current and planned ground-based instruments include the Subaru Coronagraphic Extreme Adaptive Optics instrument (Jovanovic et al. 2016), the Magellan Adaptive Optics instrument (Males et al. 2018), the Keck Planet Imager and Characterizer (Mawet et al. 2017), and the Planet Formation Imager (Monnier et al. 2018). Development continues to progress for proposed space-based imaging facilities, such as the WFIRST coronagraph (Douglas et al. 2018), the Habitable Exoplanet imaging mission (HabEx; Arya et al. 2017), and the Large Ultraviolet/Optical/Infrared Surveyor (LUVOIR; France et al. 2017). The methodology for classifying discoveries from such facilities and their expected yields is a key component for the mission science drivers (Kopparapu et al. 2018).

The observing strategy for direct imaging efforts requires an efficient target selection and time management, particularly for space-based resources. For known indirectly detected exoplanets, the optimal observing times require sufficient orbital architecture knowledge to constrain when the planet will have an angular separation from the host star that places it outside of the inner working angle (Kane 2013; Schworer \& Tuthill 2015). Many of the radial velocity (RV) planets, for example, have poorly determined orbital ephemerides due to the uncertainties in the Keplerian orbital solution compacted by the time since last observation (Kane et al. 2009; Jenkins et al. 2010). Observing those host stars after a long time baseline can help reacquire the planet's orbital phase and dramatically improve the ephemerides.

In this paper, we address the issue of determining the maximum angular separation between the star and planet for Keplerian orbital solutions. In Section 2, we discuss the challenge of orbital ephemerides and calculate the uncertainty in orbital location for 300 known exoplanets projected forward to 2025. In Section 3, we provide analytical expressions for both the star-planet separation and the derivative with respect to the true anomaly, which allows the epoch of maximum angular separation to be determined. Section 4 combines the work of the previous sections and provides calculated maximum angular separations, orbital phases where they occur, and uncertainties on those orbital locations for 50 known exoplanets. We provide concluding remarks in Section 5 and recommendations for observing strategies designed to improve orbital ephemerides for direct imaging observations.

\section{Exoplanet Orbital Ephemerides}

At the present time, exoplanet discoveries are dominated by those that utilize the transit method. Here we focus on those planets that have full Keplerian orbital solutions in order to provide a complete description of the orbital phase and angular separation. Exoplanets with Keplerian orbital solutions tend to be those discovered with the RV technique, for which survey durations have extended the period sensitivity beyond $\sim 10$ years (Wittenmyer et al. 2016). Data regarding exoplanets and orbital parameters are available from numerous sources, both in the literature and online (Butler et al. 2006; Wright et al. 2011). For this study, we utilize the data from the NASA Exoplanet Archive (Akeson et al. 2013), where the data are current as of 2018 August 17. 


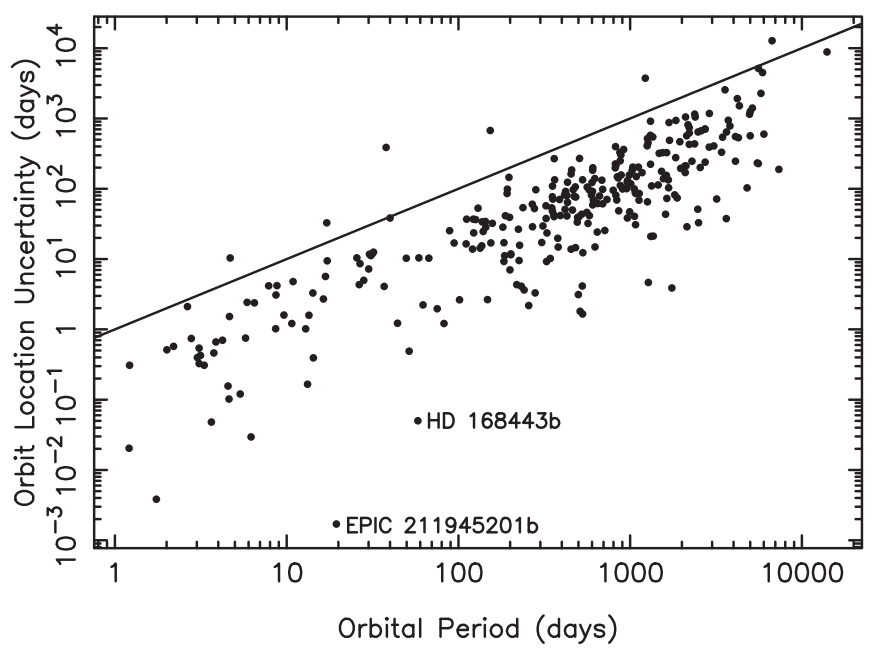

Figure 1. The calculated uncertainty in the planetary orbital location as a function of orbital period for 300 known exoplanets. The solid line shows where the uncertainty in the orbital location is the same as the orbital period. The uncertainty in the orbital location has been calculated for 2025 January 1 by propagating the uncertainties in the orbital period and time of periastron passage.

We calculate the uncertainty in the planetary orbital location for 2025 January 1 (JD =2,460,676.5). This date was chosen since it approximately matches the anticipated first light and/or launch of numerous ground and space-based telescopes that aim to directly image long-period planets. We propagate the uncertainties from the time of periastron passage using methodology of Kane et al. (2009). This methodology uses Keplerian orbital elements and their uncertainties with multiples of the orbital period to calculate epochs of specific orbit locations relative to times of measured periastron passage. Kane et al. (2009) uses this method to determine uncertainties and transit windows for times of inferior conjunction, whereas we use the method to describe the general uncertainty on orbital location. The results of these calculations are shown in Figure 1, which plots the uncertainty in the orbital location as a function of the orbital period. The relationship between these two parameters follows a power law where the uncertainty in orbital location becomes comparable to the orbital period for particularly long periods, indicated by the solid line. This means that the location of those planets in their orbit has been completely lost, making it impossible to provide useful ephemeris information for follow-up observations that require such knowledge. An example of such follow-up observations is the need to predict times of maximum angular separation for direct imaging experiments.

There are several significant outliers in Figure 1 for which the ephemerides are relatively well defined. The combination of $\mathrm{RV}$ data with transit data from the $K 2$ mission by Chakraborty et al. (2018) produced an exceptionally strong constraint on the time of periastron passage for EPIC 211945201b. In the case of HD 168443b, targeted RV observations during periastron by the Transit Ephemeris Refinement and Monitoring Survey (TERMS), combined with a long time baseline, yielded very small uncertainties on the time of periastron passage (Pilyavsky et al. 2011). In general, the calculations presented here demonstrate the need for further RV observations to reacquire the planetary location.

\section{Maximum Angular Separation}

The angular star-planet separation can be sensitive to the Keplerian orbital element of eccentricity, depending upon the orbital inclination and the argument of periastron. It is thus critical to include the full Keplerian orbital solution when calculating the angular separation.

The star-planet separation, $r$, is generally expressed as

$$
r=\frac{a\left(1-e^{2}\right)}{1+e \cos f}
$$

where $a$ is the semimajor axis, $e$ is the eccentricity, and $f$ is the true anomaly. The angular separation as a function of $f$, as computed by Kane (2013), is given by

$$
\Delta \theta=\frac{r}{d}\left(\cos ^{2}(\omega+f)+\sin ^{2}(\omega+f) \cos ^{2} i\right)^{\frac{1}{2}}
$$

where $\omega$ is the argument of periastron, $i$ is the orbital inclination, and $d$ is the star-observer distance (Kane \& Gelino 2011). When expressed in this way, the units of the angular separation in Equation (2) are in radians.

For the purposes of this work, the main objective is to determine epochs of maximum angular separation. To achieve that for a Keplerian orbit, it is necessary to substitute Equation (1) into Equation (2) and differentiate the resulting expression with respect to $f$. Such differentiation is nontrivial but does result in the following analytical expression:

$$
\begin{aligned}
\frac{d}{d f}(\Delta \theta)= & -\frac{a(e-1)^{2}}{d(e \cos f+1)^{2}} \\
& \cdot \frac{1}{\sqrt{\cos ^{2} i \sin ^{2}(\omega+f)+\cos ^{2}(\omega+f)}} \\
& \cdot\left(e \cos ^{2} i \sin f \sin ^{2}(\omega+f)+\left(\left(e \cos ^{2} i-e\right)\right.\right. \\
& \left.\cdot \cos f+\cos ^{2} i-1\right) \cos (\omega+f) \sin (\omega+f) \\
& \left.+e \sin f \cos ^{2}(\omega+f)\right) .
\end{aligned}
$$

Therefore, the maximum and minimum angular separations occur where Equation (3) equals zero (stationary points).

Shown in Figure 2 are four examples of the projected angular separation (solid line) for a hypothetical planetary system located 10 parsecs from the observer and with a planetary semimajor axis of $1 \mathrm{au}$. This is demonstrated for eccentricities of 0.2 and 0.5 and for a range of periastron arguments and orbital inclinations. An orbital phase of zero in the plots corresponds to superior conjunction where the phase angle is also zero. Also shown in Figure 2 as a dotted line is the derivative of the angular separation equation, corresponding to the rate of change of the angular separation. The stationary points (where the derivative crosses the zero-point shown as a horizontal dashed line) indicate the locations of maximum and minimum angular separations. The maximum angular separations occur at orbital phases of $0.69,0.75,0.58$, and 0.35 for the top-left, top-right, bottom-left, and bottom-right panels, respectively. It is worth noting that the maximum angular separation does not necessarily occur when the contrast ratio between the star and planet are optimal for detection, since that also depends on the phase angle and scattering properties of the atmosphere (Kane \& Gelino 2010; Nayak et al. 2017). 

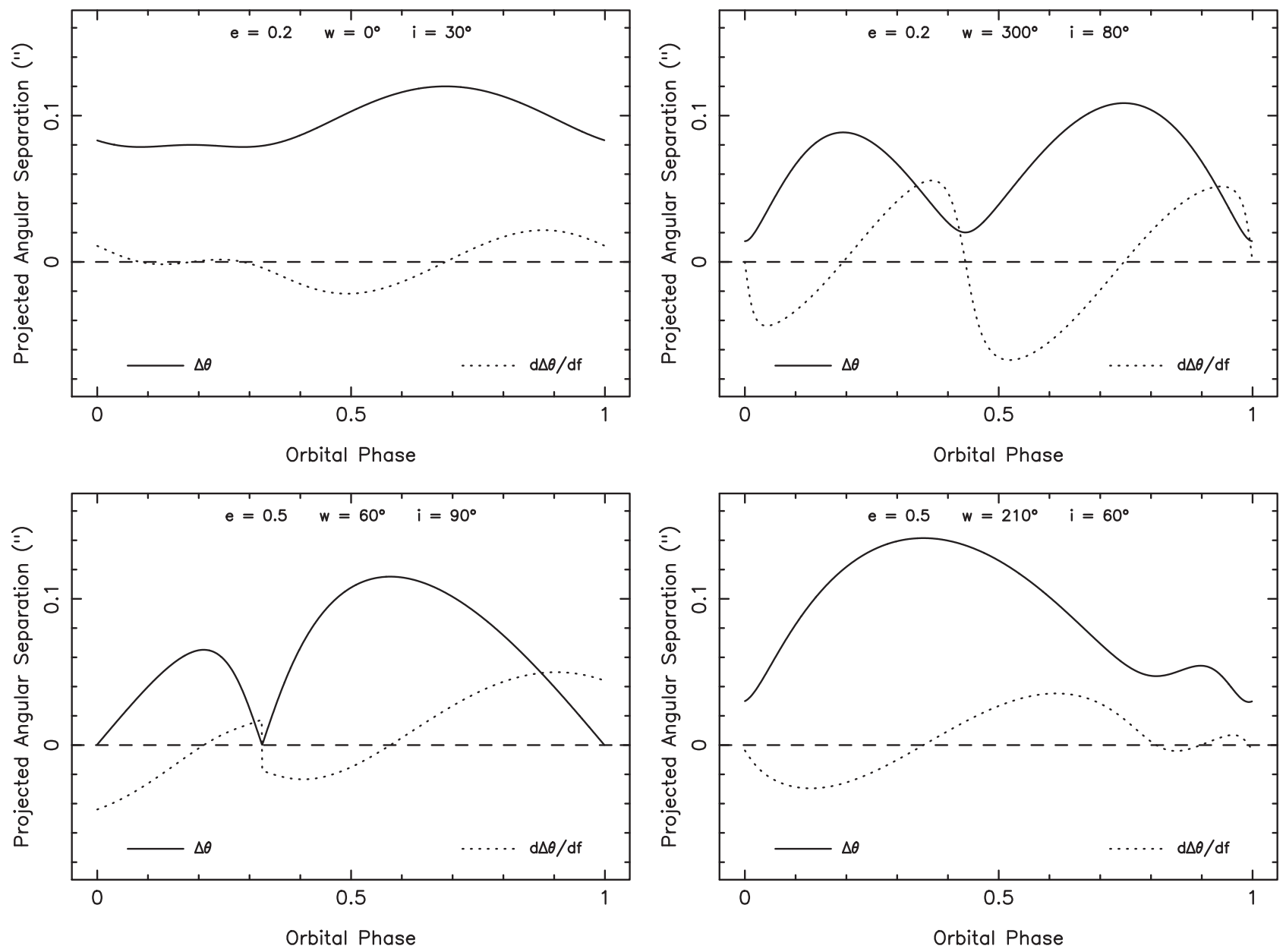

Figure 2. Projected angular separation (solid line) of a planet in a 1 au semimajor axis orbit around a star located 10 pc from the observer. The four panels shown represent a wide range of Keplerian orbital parameters, including eccentricity $e$, argument of periastron $\omega$, and orbital inclination $i$. The derivative of the angular separation is shown as a dotted line, where the intersections with the zero-point (horizontal dashed line) indicate the orbital phase locations of the minimum and maximum projected angular separation.

\section{Epochs of Optimal Observation}

Here we combine the calculations of the previous two sections and apply these to the 300 known exoplanets described in Section 2. For exoplanets without an eccentricity value, we fix the orbit to circular $(e=0.0)$. If the argument of periastron is missing, we fix the periastron to the plane perpendicular to the sky that aligns with inferior conjunction $\left(\omega=90^{\circ}\right)$. For the majority of the 300 targets considered, the inclination is unknown, and in those cases, we fix the inclination to an edgeon orientation $\left(i=90^{\circ}\right)$. This inclination was chosen as a conservative limit since approximately edge-on orbits are the most difficult for direct imaging detection.

Table 1 shows the top 50 known exoplanet targets ranked by their maximum angular separation, $\Delta \theta_{\max }$, shown in units of milliarcsecs (mas). Also included are the predicted orbital phase past superior conjunction (phase angle of zero) where the maximum angular separation will occur, $\phi_{\max }$, and the uncertainty (in orbital phase units) of when that will occur, $\sigma_{\phi}$. All of the planets represented in Table 1 are assumed to have edge-on orbits with the exception of eps Eri b, which has a measured orbital inclination of $i=30^{\circ} .1$ (Benedict et al. 2006). Therefore, the planetary masses, $M_{p}$, are minimum masses (except for eps Eri b) in units of Jupiter masses, $M_{J}$. Note that the values of $\Delta \theta_{\max }$ and $\phi_{\max }$ do not change with time (unless the orbital solution is updated), but the uncertainty in phase, $\sigma_{\phi}$, where $\Delta \theta_{\max }$ occurs does increase with time and is calculated for 2025, as described in Section 2. This means that the $\sigma_{\phi}$ values apply to the next maximum angular separation event that occurs past the 2025 date.

The highest ranked case of eps Eri b within Table 1 is represented in the panels of Figure 3. The left panel shows a top-down view of the orbit, which is highly eccentric $(e=0.7)$ and has a predicted maximum angular separation of $\Delta \theta_{\max }=1$ ". 68 . The right panel displays the angular separation as well as the rate of angular separation change (derivative of angular separation, see Equation (3)), as described in Figure 2. The combination of the large predicted maximum angular separation and the relatively small uncertainty on the orbital ephemeris ( 0.013 phase units) make this an ideal target for follow-up observations from an orbit perspective. More recent work by Mawet et al. (2018) suggests that eps Eri b has a substantially more circular orbit $(e=0.07)$, which would reduce the predicted maximum angular separation to $\Delta \theta_{\max }=1$." 14 but maintain the planet's top-ranked position in Table 1. The well-defined orbit, including orbital inclination, is a result of a simultaneous fit of RV and astrometric data through the use of Hubble Space Telescope (HST) observations (Benedict et al. 2006). Note that eps Eri is an active star that will present other observational challenges for direct detection 
Table 1

Maximum Angular Separations

\begin{tabular}{|c|c|c|c|c|c|c|c|c|c|c|}
\hline Planet & $\begin{array}{c}P \\
\text { (days) }\end{array}$ & $\begin{array}{c}a \\
(\mathrm{au})\end{array}$ & $e$ & $\begin{array}{l}\omega \\
\left(^{\circ}\right)\end{array}$ & $\begin{array}{c}i \\
\left(^{\circ}\right)\end{array}$ & $\begin{array}{c}M_{p} \\
\left(M_{J}\right)\end{array}$ & $\begin{array}{c}d \\
(\mathrm{pcs})\end{array}$ & $\begin{array}{c}\Delta \theta_{\max } \\
(\mathrm{mas})\end{array}$ & $\phi_{\max }$ & $\sigma_{\phi}$ \\
\hline eps Eri b & 2502.00 & 3.39 & 0.70 & 47.0 & 30.1 & 1.55 & 3.2 & 1679.6 & 0.609 & 0.013 \\
\hline 47 UMa d & 14002.00 & 11.60 & 0.16 & 110.0 & 90.0 & 1.64 & 14.1 & 859.6 & 0.293 & 0.630 \\
\hline HD $217107 \mathrm{c}$ & 4270.00 & 5.32 & 0.52 & 198.6 & 90.0 & 2.60 & 19.7 & 398.3 & 0.367 & 0.125 \\
\hline GJ $676 \mathrm{~A} \mathrm{c}$ & 7337.00 & 6.60 & 0.00 & 90.0 & 90.0 & 6.80 & 16.9 & 390.5 & 0.249 & 0.026 \\
\hline HD $160691 \mathrm{c}$ & 4205.80 & 5.24 & 0.10 & 57.6 & 90.0 & 1.81 & 15.3 & 359.5 & 0.719 & 0.455 \\
\hline HD 150706 b & 5894.00 & 6.70 & 0.38 & 132.0 & 90.0 & 2.71 & 27.2 & 298.6 & 0.387 & 0.762 \\
\hline HD $134987 \mathrm{c}$ & 5000.00 & 5.80 & 0.12 & 195.0 & 90.0 & 0.82 & 22.2 & 291.4 & 0.281 & 0.113 \\
\hline HD $142 \mathrm{c}$ & 6005.00 & 6.80 & 0.21 & 250.0 & 90.0 & 5.30 & 25.6 & 279.0 & 0.239 & 0.100 \\
\hline $47 \mathrm{UMa} \mathrm{c}$ & 2391.00 & 3.60 & 0.10 & 295.0 & 90.0 & 0.54 & 14.1 & 265.3 & 0.751 & 0.439 \\
\hline HD 219077 b & 5501.00 & 6.22 & 0.77 & 57.6 & 90.0 & 10.39 & 29.2 & 249.7 & 0.452 & 0.042 \\
\hline GJ 328 b & 4100.00 & 4.50 & 0.37 & 290.0 & 90.0 & 2.30 & 20.0 & 239.1 & 0.768 & 0.135 \\
\hline GJ 179 b & 2288.00 & 2.41 & 0.21 & 153.0 & 90.0 & 0.82 & 12.1 & 235.1 & 0.326 & 0.108 \\
\hline HD $166724 b$ & 5144.00 & 5.42 & 0.73 & 202.3 & 90.0 & 3.53 & 43.0 & 206.7 & 0.400 & 0.273 \\
\hline ups And d & 1276.46 & 2.51 & 0.30 & 258.8 & 90.0 & 4.13 & 13.5 & 189.2 & 0.219 & 0.004 \\
\hline HD 196067 b & 3638.00 & 5.02 & 0.66 & 148.2 & 90.0 & 6.90 & 43.6 & 172.7 & 0.499 & 0.177 \\
\hline HD $113538 \mathrm{c}$ & 1818.00 & 2.44 & 0.20 & 280.0 & 90.0 & 0.93 & 15.9 & 155.8 & 0.771 & 0.045 \\
\hline HAT-P-11 c & 3407.00 & 4.13 & 0.60 & 143.7 & 90.0 & 1.60 & 37.8 & 155.0 & 0.480 & 0.097 \\
\hline $47 \mathrm{UMa} b$ & 1078.00 & 2.10 & 0.03 & 334.0 & 90.0 & 2.53 & 14.1 & 153.4 & 0.742 & 0.080 \\
\hline nu Oph c & 3186.00 & 6.10 & 0.17 & 4.6 & 90.0 & 27.00 & 46.8 & 151.6 & 0.695 & 0.022 \\
\hline HD $106515 \mathrm{~A} \mathrm{~b}$ & 3630.00 & 4.59 & 0.57 & 123.8 & 90.0 & 9.61 & 36.4 & 151.2 & 0.459 & 0.010 \\
\hline gam Cep b & 903.30 & 2.05 & 0.05 & 94.6 & 90.0 & 1.85 & 13.8 & 149.1 & 0.259 & 0.110 \\
\hline HD 141399 e & 5000.00 & 5.00 & 0.26 & 90.0 & 90.0 & 0.66 & 36.2 & 133.4 & 0.708 & 0.251 \\
\hline HD 10647 b & 989.20 & 2.02 & 0.15 & 212.0 & 90.0 & 0.94 & 17.4 & 130.5 & 0.275 & 0.158 \\
\hline HD $220773 b$ & 3724.70 & 4.94 & 0.51 & 226.0 & 90.0 & 1.45 & 49.0 & 129.5 & 0.293 & 0.253 \\
\hline HD $133131 \mathrm{~B} \mathrm{~b}$ & 5769.00 & 6.15 & 0.61 & 103.0 & 90.0 & 2.50 & 47.0 & 123.2 & 0.413 & 0.394 \\
\hline HD 98649 b & 4951.00 & 5.60 & 0.85 & 248.0 & 90.0 & 6.80 & 42.8 & 122.3 & 0.244 & 0.232 \\
\hline HD $219828 \mathrm{c}$ & 4791.00 & 5.96 & 0.81 & 145.8 & 90.0 & 15.10 & 77.9 & 119.4 & 0.548 & 0.021 \\
\hline HD $8673 b$ & 1634.00 & 3.02 & 0.72 & 323.4 & 90.0 & 14.20 & 38.3 & 117.0 & 0.646 & 0.045 \\
\hline HD $38529 \mathrm{c}$ & 2140.20 & 3.71 & 0.34 & 17.8 & 90.0 & 13.38 & 42.4 & 115.5 & 0.627 & 0.013 \\
\hline HD $187123 \mathrm{c}$ & 3810.00 & 4.89 & 0.25 & 243.0 & 90.0 & 1.99 & 47.9 & 111.1 & 0.247 & 0.205 \\
\hline HD $160691 \mathrm{~b}$ & 643.25 & 1.50 & 0.13 & 22.0 & 90.0 & 1.08 & 15.3 & 109.5 & 0.704 & 0.038 \\
\hline HD $29021 b$ & 1362.30 & 2.28 & 0.46 & 179.5 & 90.0 & 2.40 & 30.6 & 108.7 & 0.391 & 0.016 \\
\hline HD 147513 b & 528.40 & 1.32 & 0.26 & 282.0 & 90.0 & 1.21 & 12.9 & 104.7 & 0.774 & 0.253 \\
\hline HD $169830 \mathrm{c}$ & 2102.00 & 3.60 & 0.33 & 252.0 & 90.0 & 4.04 & 36.3 & 104.2 & 0.229 & 0.499 \\
\hline HD $4203 \mathrm{c}$ & 6700.00 & 6.95 & 0.24 & 224.0 & 90.0 & 2.17 & 77.8 & 103.5 & 0.274 & 1.902 \\
\hline HD $183263 \mathrm{c}$ & 3070.00 & 4.35 & 0.24 & 345.0 & 90.0 & 3.57 & 52.8 & 101.2 & 0.688 & 0.132 \\
\hline HD $181433 d$ & 2172.00 & 3.00 & 0.48 & 90.0 & 90.0 & 0.54 & 26.1 & 100.6 & 0.325 & 0.375 \\
\hline $7 \mathrm{CMa} \mathrm{b}$ & 796.00 & 1.93 & 0.22 & 77.0 & 90.0 & 2.46 & 19.8 & 99.8 & 0.698 & 0.120 \\
\hline HD $32963 \mathrm{~b}$ & 2372.00 & 3.41 & 0.07 & 107.0 & 90.0 & 0.70 & 35.2 & 98.5 & 0.267 & 0.183 \\
\hline HD $216437 b$ & 1256.00 & 2.32 & 0.29 & 63.0 & 90.0 & 1.82 & 26.5 & 96.0 & 0.660 & 0.325 \\
\hline HD $10180 \mathrm{~h}$ & 2205.00 & 3.38 & 0.09 & 142.0 & 90.0 & 0.21 & 39.0 & 93.0 & 0.283 & 0.336 \\
\hline HD $11964 \mathrm{~b}$ & 1945.00 & 3.16 & 0.04 & 90.0 & 90.0 & 0.62 & 34.0 & 92.9 & 0.256 & 0.240 \\
\hline HD $133131 \mathrm{~A} \mathrm{c}$ & 3568.00 & 4.49 & 0.49 & 100.0 & 90.0 & 0.42 & 47.0 & 91.8 & 0.365 & 0.717 \\
\hline HD $37605 \mathrm{c}$ & 2720.00 & 3.81 & 0.01 & 221.0 & 90.0 & 3.37 & 42.9 & 89.8 & 0.251 & 0.259 \\
\hline HD $4732 \mathrm{c}$ & 2732.00 & 4.60 & 0.23 & 118.0 & 90.0 & 2.37 & 56.5 & 88.5 & 0.321 & 0.087 \\
\hline HD 79498 b & 1966.10 & 3.13 & 0.59 & 221.0 & 90.0 & 1.34 & 49.0 & 87.3 & 0.317 & 0.099 \\
\hline HAT-P-17 c & 5584.00 & 5.60 & 0.39 & 181.5 & 90.0 & 3.40 & 90.0 & 86.5 & 0.369 & 0.919 \\
\hline BD-11 4672 b & 1667.00 & 2.28 & 0.05 & 231.0 & 90.0 & 0.53 & 27.3 & 86.1 & 0.253 & 0.080 \\
\hline HD $181433 \mathrm{c}$ & 962.00 & 1.76 & 0.28 & 21.4 & 90.0 & 0.64 & 26.1 & 84.5 & 0.647 & 0.128 \\
\hline GJ 317 b & 692.00 & 1.15 & 0.11 & 342.0 & 45.0 & 2.50 & 15.1 & 84.1 & 0.717 & 0.088 \\
\hline
\end{tabular}

of the known exoplanet (Metcalfe et al. 2013; Jeffers et al. 2014).

By contrast, cases such as the 47 UMa system have large predicted maximum angular separations but relatively large uncertainties concerning when that separation will occur $(0.439$ and 0.630 phase units for the $\mathrm{c}$ and $\mathrm{d}$ planets, respectively). Such systems will benefit enormously from further RV observations at specific epochs that will provide vast improvements to the orbital solution (Kane et al. 2009). Provided that the uncertainty in orbital phase can be constrained to cover a range of orbital locations that lie outside the inner working angle of an instrumental design, the targets will be viable for observations.

\section{Conclusions}

A key component of designing imaging missions is the selection of optimal targets for observation. These are naturally drawn from the known RV exoplanets since these provide test cases for technology demonstrations and contain the necessary long-period demographic required by direct imaging experiments. The Keplerian nature of the RV orbits can lead to 

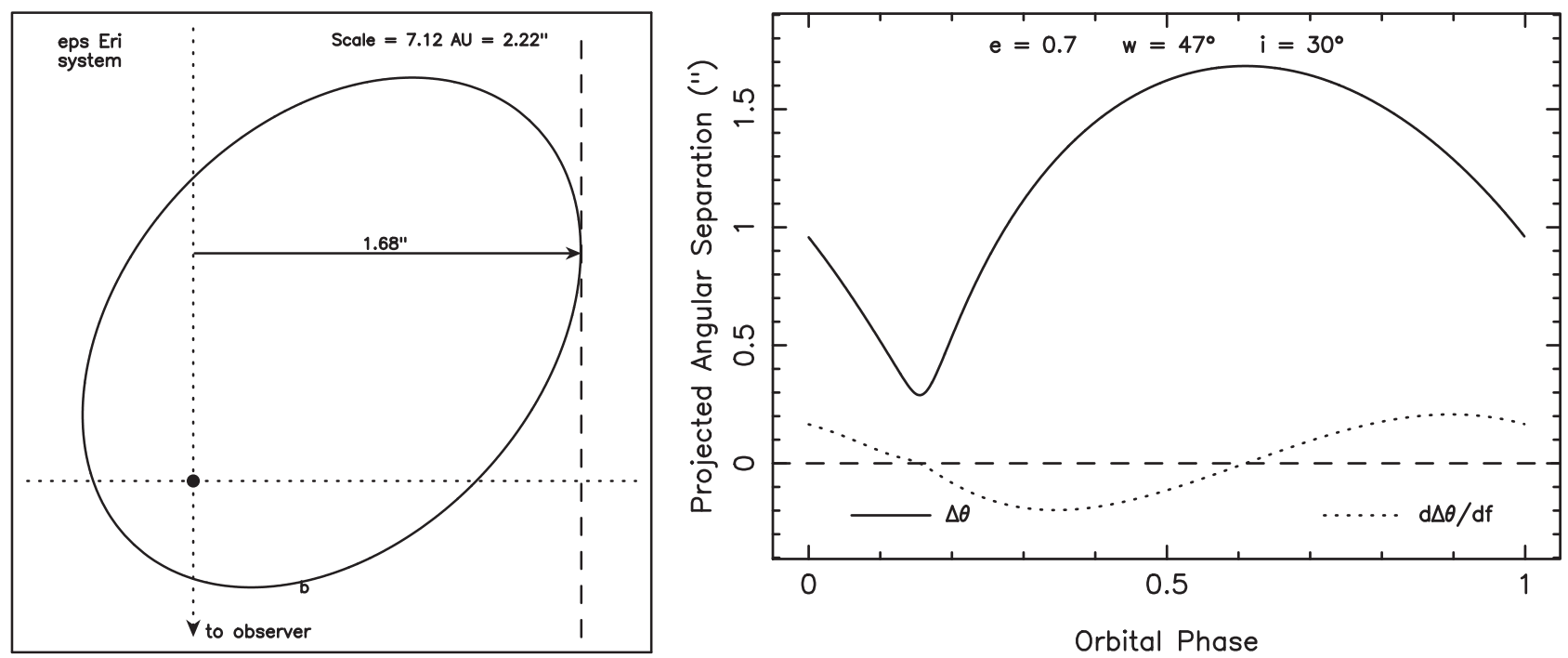

Figure 3. Plots showing the orbit and angular separation for eps Eri b. The left panel represents a top-down view of the eps Eri b orbit where the orientation with respect to the observer is shown with the dotted lines. The size of the plot one side is equivalent 7.12 au which, as the distance of the system $(d=3.21$ pcs $)$ is equivalent to an angular size of 2 ". 22 . The vertical dashed line indicates the location of maximum angular separation of the planet from the host star as seen from the observer, corresponding to the 1 .' 68 value shown in Table 1 . The right panel shows the variation in maximum angular separation (solid line) and rate of angular separation change (dotted line) for eps Eri b.

enhanced angular separations, though the timing of such separations is often poorly constrained. The methodology provided here allows the direct calculation of maximum angular separation via the stationary points of the angular separation equation. As stated at the end of Section 3, the epochs of maximum angular separation do not necessarily correspond with the epochs of expected maximum planet brightness. The contrast ratio of exoplanets depends upon numerous factors such as the wavelength of observation and also the type (terrestrial, gas giant), age, atmospheric properties, and albedo of the planet (Feng et al. 2018). The focus of this work is to allow the observation of planets that would otherwise be inside the inner working angle of the imaging experimental design (Turnbull et al. 2012).

The challenge of improving the RV targets to ensure that they will minimize telescope resources is one that must be met before a systematic imaging survey can commence. The RV time required to refine the orbits of long-period planets can be moderate, provided that one utilizes the same facility that was used to acquire the discovery data (Kane et al. 2009). Precise observing strategies depend on the properties of the individual targets and need to be customized on a case-by-case basis (Kane 2007; Bottom et al. 2013). Refining the orbits of the planets discussed in this paper will help enormously toward increasing the detection yield of missions such as WFIRST, HabEx, and LUVOIR and will also aid in planning follow-up observations with James Webb Space Telescope for detecting phase variations of known planets. For missions launching in the mid 2020s, it is paramount that the process of orbital refinement commences with sufficient lead time to avoid compromising the target list.

This research has made use of the NASA Exoplanet Archive, which is operated by the California Institute of Technology, under contract with the National Aeronautics and Space Administration under the Exoplanet Exploration Program. The results reported herein benefited from collaborations and/or information exchange within NASA's Nexus for
Exoplanet System Science (NExSS) research coordination network sponsored by NASA's Science Mission Directorate. This work was funded by the WFIRST CGI Science Investigation Team contract \#NNG16P27C (PI: Margaret Turnbull).

\section{ORCID iDs}

Stephen R. Kane iㅣ https://orcid.org/0000-0002-7084-0529 Tiffany Meshkat (i) https://orcid.org/0000-0001-6126-2467

\section{References}

Akeson, R. L., Chen, X., Ciardi, D., et al. 2013, PASP, 125, 989

Arya, M., Webb, D., McGown, J., et al. 2017, Proc. SPIE, 10400, 104001C Benedict, G. F., McArthur, B. E., Gatewood, G., et al. 2006, AJ, 132, 2206

Beuzit, J.-L., Feldt, M., Dohlen, K., et al. 2008, Proc. SPIE, 7014, 701418

Bottom, M., Muirhead, P. S., Johnson, J. A., \& Blake, C. H. 2013, PASP, 125,240

Butler, R. P., Wright, J. T., Marcy, G. W., et al. 2006, ApJ, 646, 505

Chakraborty, A., Roy, A., Sharma, R., et al. 2018, AJ, 156, 3

Cowan, N. B., Agol, E., Meadows, V. S., et al. 2009, ApJ, 700, 915

Douglas, E. S., Carlton, A. K., Cahoy, K. L., et al. 2018, Proc. SPIE, 10705 , 1070526

Feng, Y. K., Robinson, T. D., Fortney, J. J., et al. 2018, AJ, 155, 200

France, K., Fleming, B., West, G., et al. 2017, Proc. SPIE, 10397, 1039713

Jeffers, S. V., Petit, P., Marsden, S. C., et al. 2014, A\&A, 569, A79

Jenkins, J. S., Jones, H. R. A., Biller, B., et al. 2010, A\&A, 515, A17

Jovanovic, N., Guyon, O., Lozi, J., et al. 2016, Proc. SPIE, 9909, 99090W

Kane, S. R. 2007, MNRAS, 380, 1488

Kane, S. R. 2013, ApJ, 766, 10

Kane, S. R., \& Gelino, D. M. 2010, ApJ, 724, 818

Kane, S. R., \& Gelino, D. M. 2011, ApJ, 729, 74

Kane, S. R., Mahadevan, S., von Braun, K., Laughlin, G., \& Ciardi, D. R. 2009, PASP, 121, 1386

Kane, S. R., \& Torres, S. M. 2017, AJ, 154, 204

Kopparapu, R. K., Hébrard, E., Belikov, R., et al. 2018, ApJ, 856, 122

Macintosh, B., Graham, J. R., Ingraham, P., et al. 2014, PNAS, 111, 12661

Males, J. R., Close, L. M., Miller, K., et al. 2018, Proc. SPIE, 10703, 1070309

Mawet, D., Delorme, J. R., Jovanovic, N., et al. 2017, Proc. SPIE, 10400, 1040029

Mawet, D., Hirsch, L., Lee, E. J., et al. 2018, AJ, in press (arXiv:1810.03794)

Metcalfe, T. S., Buccino, A. P., Brown, B. P., et al. 2013, ApJL, 763, L26 
Monnier, J. D., Kraus, S., Irelanad, M. J., et al. 2018, ExA, in press (arXiv:1807.11559)

Nayak, M., Lupu, R., Marley, M. S., et al. 2017, PASP, 129, 034401

Pilyavsky, G., Mahadevan, S., Kane, S. R., et al. 2011, ApJ, 743, 162
Schworer, G., \& Tuthill, P. G. 2015, A\&A, 578, A59

Turnbull, M. C., Glassman, T., Roberge, A., et al. 2012, PASP, 124, 418

Wittenmyer, R. A., Butler, R. P., Tinney, C. G., et al. 2016, ApJ, 819, 28

Wright, J. T., Fakhouri, O., Marcy, G. W., et al. 2011, PASP, 123, 412 